Research Article

\title{
Global Exponential Stability of a Delayed HIV Infection Model with a Nonlinear Incidence Rate
}

\author{
Zhiwen Long (iD \\ School of Mathematics and Finance, Hunan University of Humanities, Science and Technology, Loudi 417000, Hunan, China \\ Correspondence should be addressed to Zhiwen Long; longzw2005@126.com
}

Received 24 September 2020; Revised 4 December 2020; Accepted 26 December 2020; Published 19 January 2021

Academic Editor: Rigoberto Medina

Copyright (C) 2021 Zhiwen Long. This is an open access article distributed under the Creative Commons Attribution License, which permits unrestricted use, distribution, and reproduction in any medium, provided the original work is properly cited.

Under the assumption that there is a time delay between the time target cells are contacted by the virus particles and the time the contacted cells become actively infected, we investigate the exponential stability of the noninfected equilibrium for a delayed HIV infection model with a nonlinear incidence rate. Compared with the global asymptotic stability analysis based on basic reproduction number, exponential stability analysis reveals the change range of various cells in different time periods.

\section{Introduction}

As is well-known, acquired immune deficiency syndrome (AIDS) has received widespread attention since its discovery. Initial models used to gain an insight into HIV immunology relied on systems of ordinary differential equations (see, e.g., [1-6]). In general, an underlying assumption in such an ODE model for HIV infection is that infection of cells by virions is instantaneous. In fact, in the real world, there may be intracellular delays in the viral infection and replication, and immune response processes. Furthermore, delay-differential equations exhibit much more complicated dynamics than ordinary differential equations since a time delay could affect the stability of the systems and may lead to some complex dynamic behaviors such as oscillation, chaos, and instability $[7,8]$. Hence, time delays have been incorporated into HIV infection models by some authors (see [9-16] and the references cited therein).

On the other hand, compared with bilinear incidence rate, nonlinear incidence rate usually has more complex properties. When the number of viruses and susceptible cells is large, the number of susceptible cells contacted by viruses per unit time is limited, so the nonlinear incidence rate should be adopted in this case [17]. Recently, under the assumption that there is a lag between the time target cells are contacted by the virus particles and the time the contacted cells become actively infected, Yuan et al. [18] proposed the following delay-differential system for HIV infection models with a nonlinear incidence rate:

$$
\left\{\begin{array}{l}
x^{\prime}(t)=\mu-k x(t)-\alpha x(t) f(v(t)), \\
y^{\prime}(t)=\alpha e^{-m \tau} x(t-\tau) f(v(t-\tau))-\gamma y(t)-\beta y(t) h(z(t)), \\
v^{\prime}(t)=p y(t)-d v(t), \\
z^{\prime}(t)=\delta y(t)-q z(t),
\end{array}\right.
$$

where $x(t), y(t), v(t)$, and $z(t)$ denote $C D 4^{+}$cells that are susceptible to infection, productively infected cells, virus, and the effector population of CTLs (cytotoxic $T$ lymphocytes), respectively, at time $t$; $\mu$ denotes the newly added susceptible cell, $k$ represents its death rate constant, $\alpha$ is the infection rate constant, $\gamma$ signifies the infected cell death rate constant, and $\beta$ represents the killing rate constant of productively infected cell by CTLs; $p$ denotes the rate constant of virus production by infected cell, and $d$ 
determines the clearance rate constant of virus; effectors are generated in the presence of infected cells at rate $\delta y$ and die at rate constant $q$ per cell; $\tau>0$ represents lag between the time target cells are contacted by the virus particles and the time the contacted cells become actively infected, and $e^{-m \tau}$ is assumed to describe the surviving rate constant of each target cell to get infected. The functions $f(x)$ and $h(x)$ represent the force of infection by the infective at density $x$ and the force of CTLs to kill infected cells at density $x$, respectively. Furthermore, the functions $f(x)$ and $h(x)$ are locally Lipschitz on $[0, \infty)$ and satisfy the following:

$$
\begin{aligned}
& \text { (A1) } f(0)=0, \text { the derivatives } f^{\prime}(x)>0 \text { and } \\
& (f(x) / x)^{\prime} \leq 0 \text { in }(0, \infty) \\
& \text { (A2) } h(x) \geq 0 \text { in }[0, \infty)
\end{aligned}
$$

Due to their biological relevance, all parameters in model (1) are positive. In fact, the first equation of (1) has more general form $x^{\prime}(t)=n(x(t))-g(x(t), v(t))$, where $n(x)$ is a general function that accounts for both production and turnover of healthy target cells $[19,20]$. Generally speaking, there are two main forms of this function. In addition to $n(x(t))=\lambda-d x(t) \quad[21,22]$, another typical function appearing in the literature is $n(x(t))=\lambda-d x(t)+r x(t)[1-(x(t) / K)]$, where $\lambda, d, r, K$ are positive real numbers [23, 24]. In particular, if we allow $r=0$, the second form of $n(x(t))$ will become the first one. In order to include the aforementioned two forms, we consider the following delay-differential system:

$$
\left\{\begin{array}{l}
x^{\prime}(t)=\mu-k x(t)+\rho x(t)\left[1-\frac{x(t)}{M}\right]-\alpha x(t) f(v(t)), \\
y^{\prime}(t)=\alpha e^{-m \tau} x(t-\tau) f(v(t-\tau))-\gamma y(t)-\beta y(t) h(z(t)), \\
v^{\prime}(t)=p y(t)-d v(t), \\
z^{\prime}(t)=\delta y(t)-q z(t) .
\end{array}\right.
$$

Here, $0 \leq \rho<k, \rho$ is the maximum proliferation rate of uninfected cells, and $M$ is the maximum level of uninfected cell concentration in the body. The other parameters are positive and have similar meanings to those in system (1).
For the sake of convenience, we denote by $\mathbb{R}^{n}$ the set of all $n$-dimensional real vectors. For any $x=\left(x_{1}, x_{2}, \ldots, x_{n}\right) \in \mathbb{R}^{n}$, we let $|x|$ denote the absolutevalue vector given by $|x|=\left(\left|x_{1}\right|,\left|x_{2}\right|, \ldots,\left|x_{n}\right|\right)$ and define $\|x\|=\max _{i \in\{1,2, \ldots, n\}}\left|x_{i}\right|$. Let $\mathbb{R}_{+}$denote nonnegative real number space, $C=C([-\tau, 0], \mathbb{R})$ be the Banach space of continuous functions mapping the interval $[-\tau, 0]$ into $\mathbb{R}$ equipped with the usual supremum norm $\|\cdot\|$, and let $C_{+}=C\left([-\tau, 0], \mathbb{R}_{+}\right)$. Set $x_{t}(\theta)=x(t+\theta)$ for all $\theta \in[-\tau, 0]$.

From the biological meanings, the initial conditions associated with (2) are defined as follows:

$$
x(\theta)=\varphi(\theta), v(\theta)=\psi(\theta), \quad y(0), z(0) \in \mathbb{R}_{+}, \theta \in[-\tau, 0],
$$

where $\varphi, \psi \in C\left([-\tau, 0], \mathbb{R}_{+}\right)$.

Most recently, by using the characteristic equation and the Fluctuation lemma, Yuan et al. [18] proved the following result:

Theorem 1. Let the basic reproduction number

$$
R_{0}=\frac{\alpha p \mu e^{-m \tau} f^{\prime}(0)}{k \gamma d}<1 .
$$

Then, the noninfected equilibrium $E^{0}=((\mu / k), 0,0,0)$ of (1) is globally asymptotically stable.

However, Theorem 1 does not give us any information about the convergence rate, which is vitally important to the disease prevention and control in real-world applications of theoretical results on epidemic models. In fact, the known convergence rate means that the range of the population is predictable; that is to say, we can estimate the range of changes in the population within a given time range. In particular, since the exponential convergence rate reveals the variation range of population in different time periods, there have been extensive results on the problem of the exponential stability of epidemic models in the literature studies [25-28]. Now, a question naturally arises: under what conditions is the noninfected equilibrium $\left(x^{*}, y^{*}, v^{*}, z^{*}\right)=$ $\left(x^{*}(\rho), 0,0,0\right)$ of system (2) with initial conditions (3) are exponentially stable? Here,

$$
x^{*}(\rho)=\frac{M(\rho-k)+\left(M^{2}(\rho-k)^{2}+4 \rho M \mu\right)^{1 / 2}}{2 \rho}, \quad \rho>0 ; x^{*}(0)=\frac{\mu}{k}, \rho=0 .
$$

It is easy to see that $\lim _{\rho \longrightarrow 0^{+}} x^{*}(\rho)=x^{*}(0)=(\mu / k)$. For $0 \leq \rho<k$, we denote $x^{*}(\rho)$ by $x^{*}$ for simplicity of notation.
Motivated by the above discussions, the main purpose of this paper is to establish sufficient conditions for the 
exponential stability of the noninfected equilibrium $\left(x^{*}, y^{*}, v^{*}, z^{*}\right)=\left(x^{*}, 0,0,0\right)$ of system (2). To the best of our knowledge, it is the first time to focus on the problem of the exponential stability for (2). In particular, a numerical example is provided to illustrate our theoretical results.

Similar to the reference [29], we give the definition of the exponential stability as follows.

Definition 1. Let $(x(t), y(t), v(t), z(t))$ be the solution of (2) with initial value conditions (3). If there exists a positive constant $\lambda>0$ such that

$$
\begin{aligned}
\left|x(t)-x^{*}\right| & =O\left(e^{-\lambda t}\right), \\
\left|y(t)-y^{*}\right| & =O\left(e^{-\lambda t}\right), \\
\left|v(t)-v^{*}\right| & =O\left(e^{-\lambda t}\right), \\
\left|z(t)-z^{*}\right| & =O\left(e^{-\lambda t}\right),
\end{aligned}
$$

as $t \longrightarrow+\infty$, then the noninfected equilibrium is said to be globally exponentially stable.

In the rest of the paper, we give our main result in Section 2. The theoretical result is illustrated with examples as well as numerical simulations in Section 3. Finally, conclusions are made in Section 4.

\section{Main Results}

By the fundamental theory of functional differential equations [30], we have that there is a unique solution $(x(t), y(t), v(t), z(t))$ satisfying system (2) with initial conditions (3). Furthermore, by a similar argument as that in Zhu et al. [31], it is not hard to show that every solution of (2) with initial value conditions (3) is nonnegative and bounded on $[0, \infty)$. From (2), we have

$$
x^{\prime}(t) \leq \mu-(k-\rho) x(t)
$$

which implies

$$
\begin{aligned}
x(t) & \leq x(0) e^{-(k-\rho) t}+\frac{\mu\left[1-e^{-(k-\rho) t}\right]}{k-\rho} \\
& =\frac{\mu}{k-\rho}+\left[x(0)-\frac{\mu}{k-\rho}\right] e^{-(k-\rho) t},
\end{aligned}
$$

for all $t \in(0, \infty)$.

In addition, from (A1), we can get

$$
f(\xi) \leq f^{\prime}(0) \xi, \quad \xi \in(0, \infty) .
$$

The following theorem is our main result.

Theorem 2. Assume that the following

$$
\left\{\alpha f^{\prime}(0)<\min \left\{\frac{k-\rho+\left(\rho x^{*} / M\right)-(\rho \mu / M(k-\rho))}{x^{*}}, \frac{\gamma e^{m \tau}(k-\rho)}{\mu}\right\}, \quad p<d, \delta<q\right.
$$

hold. Then, there exist three positive constants $\lambda, K$, and $t_{\xi} \quad$ for all $t>t_{\xi}$. Here, $\left(x^{*}, y^{*}, v^{*}, z^{*}\right)=\left(x^{*}(\rho), 0,0,0\right)$, and such that

$$
\begin{aligned}
\left|x(t)-x^{*}\right| & \leq K e^{-\lambda t}, \\
\left|y(t)-y^{*}\right| & \leq K e^{-\lambda t}, \\
\left|v(t)-v^{*}\right| & \leq K e^{-\lambda t}, \\
\left|z(t)-z^{*}\right| & \leq K e^{-\lambda t},
\end{aligned}
$$

$$
x^{*}(\rho)=\frac{M(\rho-k)+\left(M^{2}(\rho-k)^{2}+4 \rho M \mu\right)^{(1 / 2)}}{2 \rho}, \quad \rho>0 ; x^{*}(0)=\frac{\mu}{k}, \rho=0 .
$$


Proof. Let

$$
\begin{aligned}
X(t) & =\left(x_{1}(t), x_{2}(t), x_{3}(t), x_{4}(t)\right) \\
& =\left(x(t)-x^{*}, y(t)-0, v(t)-0, z(t)-0\right),
\end{aligned}
$$

$$
\begin{aligned}
& \left\{\begin{array}{l}
x_{1}^{\prime}(t)=-k x_{1}(t)+\rho x_{1}(t)-\frac{\rho}{M} x^{*} x_{1}(t)-\frac{\rho}{M} x(t) x_{1}(t)-\alpha x_{1}(t) f\left(x_{3}(t)\right)-\alpha x^{*} f\left(x_{3}(t)\right), \\
x_{2}^{\prime}(t)=\alpha e^{-m \tau} x(t-\tau) f\left(x_{3}(t-\tau)\right)-\gamma x_{2}(t)-\beta x_{2}(t) h\left(x_{4}(t)\right), \\
x_{3}^{\prime}(t)=p x_{2}(t)-d x_{3}(t), \\
x_{4}^{\prime}(t)=\delta x_{2}(t)-q x_{4}(t),
\end{array}\right.
\end{aligned}
$$

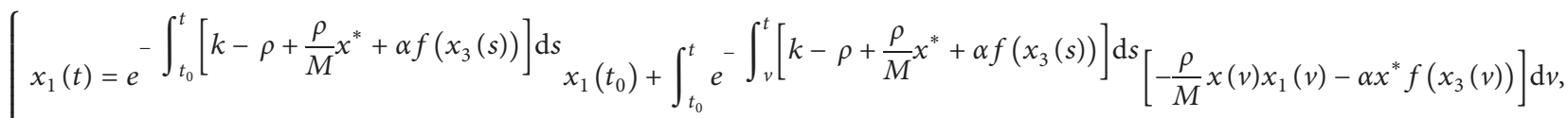

$$
\begin{aligned}
& \left\{\begin{array}{l}
x_{2}(t)=e^{-\int_{t_{0}}^{t}\left[\gamma+\beta h\left(x_{4}(s)\right)\right] \mathrm{d} s} x_{2}\left(t_{0}\right)+\int_{t_{0}}^{t} e^{-\int_{v}^{t}\left[\gamma+\beta h\left(x_{4}(s)\right)\right] \mathrm{d} s} \alpha e^{-m \tau} x(v-\tau) f\left(x_{3}(v-\tau)\right) \mathrm{d} v, \\
x_{3}(t)=e^{-d\left(t-t_{0}\right)} x_{3}\left(t_{0}\right)+\int_{t_{0}}^{t} e^{-d(t-v)} p x_{2}(v) \mathrm{d} v
\end{array}\right. \\
& x_{4}(t)=e^{-q\left(t-t_{0}\right)} x_{4}\left(t_{0}\right)+\int_{t_{0}}^{t} e^{-q(t-v)} \delta x_{2}(v) \mathrm{d} v,
\end{aligned}
$$

for all $t \geq t_{0}$.

From (8) and (10), there exist $t_{*}>0$ and $\varepsilon_{0}>0$ such that

$$
\begin{gathered}
x(t) \leq \varepsilon_{0}+\frac{\mu}{k-\rho}, \text { for all } t \in\left(t_{*}, \infty\right), \\
\alpha e^{-m \tau} f^{\prime}(0)\left(\frac{\mu}{k-\rho}+\varepsilon_{0}\right)<\gamma .
\end{gathered}
$$

Then, for any $\varepsilon \in\left(0, \varepsilon_{0}\right]$, we can choose two positive constants $\lambda$ and $\eta$ such that

$$
\left\{\begin{array}{l}
\lambda+\frac{\rho}{M}\left(\frac{\mu}{k-\rho}+\varepsilon\right)+\alpha x^{*} f^{\prime}(0)-\left(k-\rho+\frac{\rho}{M} x^{*}\right)<-\eta<0, \\
\lambda+\alpha e^{\lambda \tau} e^{-m \tau} f^{\prime}(0)\left(\frac{\mu}{k-\rho}+\varepsilon\right)-\gamma<-\eta<0, \\
\lambda+p-d<-\eta<0, \\
\lambda+\delta-q<-\eta<0 .
\end{array}\right.
$$

For a given $\varepsilon \in\left(0, \varepsilon_{0}\right]$, from (8), we can find $t_{1}>0$ such that

$$
x(t) \leq \varepsilon+\frac{\mu}{k-\rho}, \quad \text { for all } t \in\left(t_{1}, \infty\right) .
$$

Let $t_{\xi}=t_{1}+\tau, K_{\varphi}>1$, and

$$
\|X\|_{\xi}=\max \left\{\max _{s \in\left[t_{0}-\tau, t_{\xi}\right]}\left|x_{1}(s)\right|, \max _{s \in\left[t_{0}-\tau, t_{\xi}\right]}\left|x_{2}(s)\right|, \max _{s \in\left[t_{0}-\tau, t_{\xi}\right]}\left|x_{3}(s)\right|, \max _{s \in\left[t_{0}-\tau, t_{\xi}\right]}\left|x_{4}(s)\right|\right\} .
$$


Consequently,

$\left\|X\left(t_{\xi}\right)\right\|<\|X\|_{\xi}+\varepsilon<K_{\varphi}\left(\|X\|_{\xi}+\varepsilon\right)=K_{\varphi}\left(\|X\|_{\xi}+\varepsilon\right) e^{\lambda t_{\xi}} e^{-\lambda t_{\xi}}$.

In the following, we will show

$$
\|X(t)\|<K_{\varphi}\left(\|X\|_{\xi}+\varepsilon\right) e^{\lambda t_{\xi}} e^{-\lambda t}, \quad \text { for all } t>t_{\xi} .
$$

If not, one of the following four cases must occur.

Case I: there exists $\theta_{1}>0$ such that

$$
\left\{\begin{array}{l}
\left|x_{1}\left(\theta_{1}\right)\right|=K_{\varphi}\left(\|X\|_{\xi}+\varepsilon\right) e^{\lambda t_{\xi}} e^{-\lambda \theta_{1}}, \\
\|X(t)\|<K_{\varphi}\left(\|X\|_{\xi}+\varepsilon\right) e^{\lambda t_{\xi}} e^{-\lambda t}, \quad \text { for all } t \in\left[t_{0}-\tau, \theta_{1}\right) .
\end{array}\right.
$$

Case II: there exists $\theta_{2}>0$ such that

$$
\left\{\begin{array}{l}
\left|x_{2}\left(\theta_{2}\right)\right|=K_{\varphi}\left(\|X\|_{\xi}+\varepsilon\right) e^{\lambda t_{\xi}} e^{-\lambda \theta_{2}}, \\
\|X(t)\|<K_{\varphi}\left(\|X\|_{\xi}+\varepsilon\right) e^{\lambda t_{\xi}} e^{-\lambda t}, \quad \text { for all } t \in\left[t_{0}-\tau, \theta_{2}\right) .
\end{array}\right.
$$

Case III: there exists $\theta_{3}>0$ such that

$$
\left\{\begin{array}{l}
\left|x_{3}\left(\theta_{3}\right)\right|=K_{\varphi}\left(\|X\|_{\xi}+\varepsilon\right) e^{\lambda t_{\xi}} e^{-\lambda \theta_{3}}, \\
\|X(t)\|<K_{\varphi}\left(\|X\|_{\xi}+\varepsilon\right) e^{\lambda t_{\xi}} e^{-\lambda t}, \quad \text { for all } t \in\left[t_{0}-\tau, \theta_{3}\right) .
\end{array}\right.
$$

Case IV: there exists $\theta_{4}>0$ such that

$$
\left\{\begin{array}{l}
\left|x_{4}\left(\theta_{4}\right)\right|=K_{\varphi}\left(\|X\|_{\xi}+\varepsilon\right) e^{\lambda t_{\xi}} e^{-\lambda \theta_{4}}, \\
\|X(t)\|<K_{\varphi}\left(\|X\|_{\xi}+\varepsilon\right) e^{\lambda t_{\xi}} e^{-\lambda t}, \quad \text { for all } t \in\left[t_{0}-\tau, \theta_{4}\right) .
\end{array}\right.
$$

If Case I holds, in view of (9), (14), (16), (17) and (21), we have

$$
\begin{aligned}
& \left|x_{1}\left(\theta_{1}\right)\right|=\left|e^{-\int_{t_{\xi}}^{\theta_{1}}\left[k-\rho+\frac{\rho}{M} x^{*}+\alpha f\left(x_{3}(s)\right)\right] \mathrm{d} s} x_{1}\left(t_{\xi}\right)+\int_{t_{\xi}}^{\theta_{1}} e^{-\int_{v}^{\theta_{1}}\left[k-\rho+\frac{\rho}{M} x^{*}+\alpha f\left(x_{3}(s)\right)\right] \mathrm{d} s}\left[-\frac{\rho}{M} x(v) x_{1}(v)-\alpha x^{*} f\left(x_{3}(v)\right)\right] \mathrm{d} v\right| \\
& \leq e^{-\left(k-\rho+\frac{\rho}{M} x^{*}\right)\left(\theta_{1}-t_{\xi}\right)}\left|x_{1}\left(t_{\xi}\right)\right|+\int_{t_{\xi}}^{\theta_{1}} e^{-\left(k-\rho+\frac{\rho}{M} x^{*}\right)\left(\theta_{1}-v\right)}\left[\frac{\rho}{M}\left(\frac{\mu}{k-\rho}+\varepsilon\right) x_{1}(v)+\alpha x^{*} f^{\prime}(0) x_{3}(v)\right] \mathrm{d} v \\
& \leq e^{-\left(k-\rho+\frac{\rho}{M} x^{*}\right)\left(\theta_{1}-t_{\xi}\right)}\left(\|X\|_{\xi}+\varepsilon\right)+\int_{t_{\xi}}^{\theta_{1}} e^{-\left(k-\rho+\frac{\rho}{M} x^{*}\right)\left(\theta_{1}-v\right)}\left[\frac{\rho}{M}\left(\frac{\mu}{k-\rho}+\varepsilon\right)+\alpha x^{*} f^{\prime}(0)\right] K_{\varphi}\left(\|X\|_{\xi}+\varepsilon\right) e^{\lambda t_{\xi}} e^{-\lambda v} \mathrm{~d} v \\
& \leq K_{\varphi}\left(\|X\|_{\xi}+\varepsilon\right) e^{\lambda t_{\xi}} e^{-\lambda \theta_{1}}\left\{\frac{1}{K_{\varphi}} e^{-\left(\theta_{1}-t_{\xi}\right)\left(k-\rho+\frac{\rho}{M} x^{*}-\lambda\right)}+\int_{t_{\xi}}^{\theta_{1}} e^{-\left(\theta_{1}-v\right)\left(k-\rho+\frac{\rho}{M} x^{*}-\lambda\right)}\left(\frac{\rho}{M}\left(\frac{\mu}{k-\rho}+\varepsilon\right)+\alpha x^{*} f^{\prime}(0)\right) \mathrm{d} v\right\} \\
& \leq K_{\varphi}\left(\|X\|_{\xi}+\varepsilon\right) e^{\lambda t_{\xi}} e^{-\lambda \theta_{1}}\left\{\frac{1}{K_{\varphi}} e^{-\left(\theta_{1}-t_{\xi}\right)\left(k-\rho+\frac{\rho}{M} x^{*}-\lambda\right)}+\int_{t_{\xi}}^{\theta_{1}} e^{-\left(\theta_{1}-v\right)\left(k-\rho+\frac{\rho}{M} x^{*}-\lambda\right)}\left(k-\rho+\frac{\rho}{M} x^{*}-\lambda\right) \mathrm{d} v\right\} \\
& =K_{\varphi}\left(\|X\|_{\xi}+\varepsilon\right) e^{\lambda t_{\xi}} e^{-\lambda \theta_{1}}\left\{1-\left(1-\frac{1}{K_{\varphi}}\right) e^{-\left(\theta_{1}-t_{\xi}\right)\left(k-\rho+\frac{\rho}{M} x^{*}-\lambda\right)}\right\} \\
& <K_{\varphi}\left(\|X\|_{\xi}+\varepsilon\right) e^{\lambda t_{\xi}} e^{-\lambda \theta_{1}},
\end{aligned}
$$

which contradicts with the first equation in (21). Hence, Case I could not hold.
If Case II holds, combining (A2), (9), (14), (16) and (17), and (22), we deduce that 


$$
\begin{aligned}
& \left|x_{2}\left(\theta_{2}\right)\right|=\left|e^{-\int_{t_{\xi}}^{\theta_{2}}\left[\gamma+\beta h\left(x_{4}(s)\right)\right] \mathrm{d} s} x_{2}\left(t_{\xi}\right)+\int_{t_{\xi}}^{\theta_{2}} e^{-\int_{v}^{\theta_{2}}\left[\gamma+\beta h\left(x_{4}(s)\right)\right] \mathrm{d} s}\left[\alpha e^{-m \tau} x(v-\tau) f\left(x_{3}(v-\tau)\right)\right] \mathrm{d} v\right| \\
& \leq e^{-\left(\theta_{2}-t_{\xi}\right) \gamma}\left|x_{2}\left(t_{\xi}\right)\right|+\int_{t_{\xi}}^{\theta_{2}} e^{-\left(\theta_{2}-v\right) \gamma}\left[\alpha e^{-m \tau} f^{\prime}(0)\left(\frac{\mu}{k-\rho}+\varepsilon\right)\left|x_{3}(v-\tau)\right|\right] \mathrm{d} v \\
& =e^{-\left(\theta_{2}-t_{\xi}\right) \gamma}\left(\|X\|_{\xi}+\varepsilon\right)+\int_{t_{\xi}}^{\theta_{2}} e^{-\left(\theta_{2}-v\right) \gamma}\left[\alpha e^{-m \tau} f^{\prime}(0)\left(\frac{\mu}{k-\rho}+\varepsilon\right)\right] K_{\varphi}\left(\|X\|_{\xi}+\varepsilon\right) e^{\lambda t_{\xi}} e^{-\lambda(v-\tau)} \mathrm{d} v \\
& \leq K_{\varphi}\left(\|X\|_{\xi}+\varepsilon\right) e^{\lambda t_{\xi}} e^{-\lambda \theta_{2}}\left\{\frac{1}{K_{\varphi}} e^{-\left(\theta_{2}-t_{\xi}\right)(\gamma-\lambda)}+\int_{t_{\xi}}^{\theta_{2}} e^{-\left(\theta_{2}-v\right)(\gamma-\lambda)}(\gamma-\lambda) \mathrm{d} v\right\} \\
& =K_{\varphi}\left(\|X\|_{\xi}+\varepsilon\right) e^{\lambda t_{\xi}} e^{-\lambda \theta_{2}}\left\{1-\left(1-\frac{1}{K_{\varphi}}\right) e^{-\left(\theta_{2}-t_{\xi}\right)(\gamma-\lambda)}\right\} \\
& <K_{\varphi}\left(\|X\|_{\xi}+\varepsilon\right) e^{\lambda t_{\xi}} e^{-\lambda \theta_{2}},
\end{aligned}
$$

which contradicts the first equation in (22). Thus, (22) could not hold.

$$
\begin{aligned}
\left|x_{3}\left(\theta_{3}\right)\right| & =\left|e^{-d\left(\theta_{3}-t_{\xi}\right)} x_{3}\left(t_{\xi}\right)+\int_{t_{\xi}}^{\theta_{3}} p x_{2}(s) e^{-d\left(\theta_{3}-s\right)} \mathrm{d} s\right| \\
& \leq e^{-d\left(\theta_{3}-t_{\xi}\right)}\left(\|X\|_{\xi}+\varepsilon\right)+\int_{t_{\xi}}^{\theta_{3}} p e^{-d\left(\theta_{3}-s\right)} K_{\varphi}\left(\|X\|_{\xi}+\varepsilon\right) e^{\lambda t_{\xi}} e^{-\lambda s} \mathrm{~d} s \\
& \leq K_{\varphi}\left(\|X\|_{\xi}+\varepsilon\right) e^{\lambda t_{\xi}} e^{-\lambda \theta_{3}}\left\{\frac{1}{K_{\varphi}} e^{-\left(\theta_{3}-t_{\xi}\right)(d-\lambda)}+\int_{t_{\xi}}^{\theta_{3}} e^{-\left(\theta_{3}-s\right)(d-\lambda)} p \mathrm{~d} s\right\} \\
& \leq K_{\varphi}\left(\|X\|_{\xi}+\varepsilon\right) e^{\lambda t_{\xi}} e^{-\lambda \theta_{3}}\left\{\frac{1}{K_{\varphi}} e^{-\left(\theta_{3}-t_{\xi}\right)(d-\lambda)}+\int_{t_{\xi}}^{\theta_{3}} e^{-\left(\theta_{3}-s\right)(d-\lambda)}(d-\lambda) \mathrm{d} s\right\} \\
& =K_{\varphi}\left(\|X\|_{\xi}+\varepsilon\right) e^{\lambda t_{\xi}} e^{-\lambda \theta_{3}}\left\{1-\left(1-\frac{1}{K_{\varphi}}\right) e^{-\left(\theta_{3}-t_{\xi}\right)(d-\lambda)}\right\} \\
& <K_{\varphi}\left(\|X\|_{\xi}+\varepsilon\right) e^{\lambda t_{\xi}} e^{-\lambda \theta_{3}},
\end{aligned}
$$


which contradicts the first equation in (23). Thus, (23) could

If Case IV holds, together with (14) and (16), (24) yields not hold.

$$
\begin{aligned}
\left|x_{4}\left(\theta_{4}\right)\right| & =\mid e^{-q\left(\theta_{4}-t_{\xi}\right)} x_{4}\left(t_{\xi}\right)+\int_{t_{\xi}}^{\theta_{4}} \delta x_{2}(s) e^{-q\left(\theta_{4}-s\right)} \mathrm{d} s \\
& \leq e^{-q\left(\theta_{4}-t_{\xi}\right)}\left(\|X\|_{\xi}+\varepsilon\right)+\int_{t_{\xi}}^{\theta_{4}} e^{-q\left(\theta_{4}-s\right)} \delta K_{\varphi}\left(\|X\|_{\xi}+\varepsilon\right) e^{\lambda t_{\xi}} e^{-\lambda s} \mathrm{~d} s \\
& \leq K_{\varphi}\left(\|X\|_{\xi}+\varepsilon\right) e^{\lambda t_{\xi}} e^{-\lambda \theta_{4}}\left\{\frac{1}{K_{\varphi}} e^{-\left(\theta_{4}-t_{\xi}\right)(q-\lambda)}+\int_{t_{\xi}}^{\theta_{4}} e^{-\left(\theta_{4}-s\right)(q-\lambda)} \delta \mathrm{d} s\right\} \\
& \leq K_{\varphi}\left(\|X\|_{\xi}+\varepsilon\right) e^{\lambda t_{\xi}} e^{-\lambda \theta_{4}}\left\{\frac{1}{K_{\varphi}} e^{-\left(\theta_{4}-t_{\xi}\right)(q-\lambda)}+\int_{t_{\xi}}^{\theta_{4}} e^{-\left(\theta_{4}-s\right)(q-\lambda)}(q-\lambda) \mathrm{d} s\right\} \\
& =K_{\varphi}\left(\|X\|_{\xi}+\varepsilon\right) e^{\lambda t_{\xi}} e^{-\lambda \theta_{4}}\left\{1-\left(1-\frac{1}{K_{\varphi}}\right) e^{-\left(\theta_{4}-t_{\xi}\right)(q-\lambda)}\right\} \\
& <K_{\varphi}\left(\|X\|_{\xi}+\varepsilon\right) e^{\lambda t_{\xi}} e^{-\lambda \theta_{4}},
\end{aligned}
$$

which contradicts with the first equation in (24). Thus, (24) could not hold.

Therefore, we obtain from the above discussions that

$$
\|X(t)\|<K_{\varphi}\left(\|X\|_{\xi}+\varepsilon\right) e^{\lambda t_{\xi}} e^{-\lambda t}, \quad \text { for all } t>t_{\xi},
$$

holds. Letting $\varepsilon \longrightarrow 0^{+}$, we have that

$$
\|X(t)\| \leq K_{\varphi}\|X\|_{\xi} e^{\lambda t_{\xi}} e^{-\lambda t}, \quad \text { for all } t>t_{\xi},
$$

which proves (11), where $K=K_{\varphi}\|X\|_{\xi} e^{\lambda t_{\xi}}$. This completes the proof of Theorem 2 .

According to the above discussion, it is easy to see that our result is also true when $\rho=0$. Furthermore, compared with Theorem 1, we find that the significantly stronger conclusion of Theorem 2 is obtained with only slightly stricter conditions.

\section{A Numerical Example}

In this section, we will show the existence and global exponential stability of the noninfected equilibrium of system (2) by a numerical example.

Let $\mu=24, k=0.3, \rho=0.05, M=80, \alpha=0.01, m=2$, $\tau=1, \gamma=0.25, \beta=0.03, p=0.02, d=0.05, \delta=0.04, q=$ $0.07, f(x)=0.2 x$, and $h(x)=x+0.5 \sin x$. Then,

$$
\begin{aligned}
& x^{*}(\rho)=\frac{M(\rho-k)+\left(M^{2}(\rho-k)^{2}+4 \rho M \mu\right)^{1 / 2}}{2 \rho}, \quad \rho>0 ; x^{*}(0)=80 \\
& \alpha f^{\prime}(0)=0.002<0.003=\min \left\{\frac{k-\rho+\left(\rho x^{*} / M\right)-(\rho \mu / M(k-\rho))}{x^{*}}, \frac{\gamma e^{m \tau}(k-\rho)}{\mu}\right\} \\
& p=0.02<d=0.05 \\
& \delta=0.04<q=0.07 \\
&\left\{\begin{array}{l}
x^{\prime}(t)=24-0.3 x(t)+0.05 x(t)\left[1-\frac{x(t)}{80}\right]-0.002 x(t) v(t) \\
y^{\prime}(t)=0.002 e^{-2} x(t-1) v(t-1)-0.25 y(t)-0.03 y(t)[z(t)+0.5 \sin (z(t))] \\
v^{\prime}(t)=0.02 y(t)-0.05 v(t) \\
z^{\prime}(t)=0.04 y(t)-0.07 z(t)
\end{array}\right.
\end{aligned}
$$

which is globally exponentially stable, and all solutions of system (31) converge exponentially to the noninfected equilibrium
$(80,0,0,0)$ with the exponential convergent rate $\zeta \approx 0.002$. This fact is verified by the numerical simulation in Figure 1. 


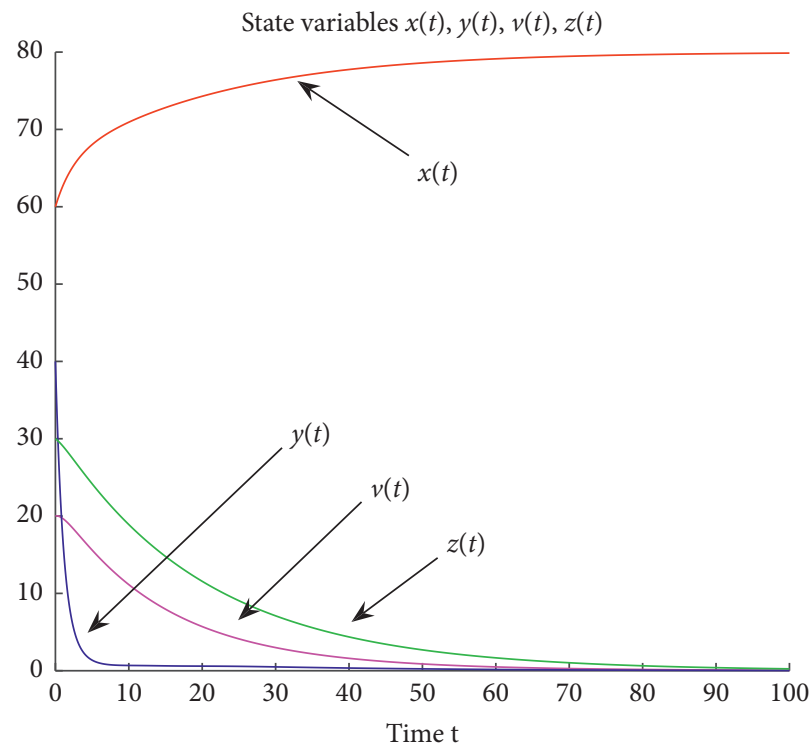

(a)

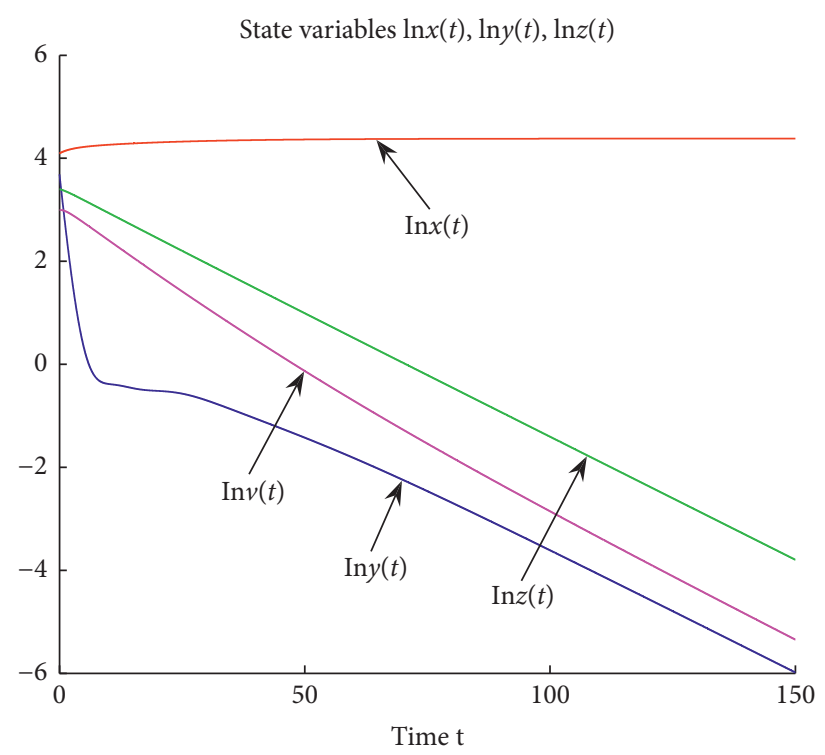

(b)

Figure 1: (a) The numerical solution $(x(t), y(t), v(t), z(t))$ of equation (31) for the initial values $x_{s} \equiv 60, y(0)=40, v_{s} \equiv 30, z(0)=20, s \in[-1,0]$; (b) the numerical simulation of logarithmic solution $(x(t), y(t), v(t), z(t))$ of equation $(31)$.

Since the results in $[3,4,19,32,33]$ give no opinions about the global exponential stability of the HIV infection models, it is clear that all the results in the above references cannot be applicable to prove the global exponential stability of system (31).

\section{Conclusion}

We have proved the global exponential stability of the noninfected equilibrium for a delayed HIV infection model with a nonlinear incidence rate. It is worth pointing out that the required conditions are simple and easy to verify. It is natural to ask whether our methods in this paper are available to study the global exponential stability of the infected equilibrium of the delayed HIV infection models. It is an issue worth our further study. In addition, as pointed out in the literature $[34,35]$, the further extension of the model is to consider the case with reaction-diffusion term, which is also the focus of our further research.

\section{Data Availability}

The data used to support the findings of this study are included within the article.

\section{Conflicts of Interest}

The authors declare that they have no conflicts of interest.

\section{Acknowledgments}

This work was jointly supported by Natural Scientific Research Fund of Hunan Province (2018JJ2194) and Scientific Research Fund of Hunan Provincial Education Department of China (18B456, 19C0985, 19C0976), the construct program of the applied characteristic discipline "Applied Economics" in Hunan Province.

\section{References}

[1] A. S. Perelson and P. W. Nelson, "Mathematical analysis of HIV-1 dynamics in vivo," Siam Review, vol. 41, no. 1, pp. 3-44, 1999.

[2] M. S. Ciupe, B. L. Bivort, D. M. Bortz, and P. W. Nelson, "Estimating kinetic parameters from HIV primary infection data through the eyes of three different mathematical models," Mathematical Biosciences, vol. 200, no. 1, pp. 1-27, 2006.

[3] L. Duan and Z. Xu, "A note on the dynamics analysis of a diffusive cholera epidemic model with nonlinear incidence rate," Applied Mathematics Letters, vol. 106, Article ID 106356, 2020.

[4] L. Rong and A. S. Perelson, "Modeling HIV persistence, the latent reservoir, and viral blips," Journal of Theoretical Biology, vol. 260, no. 2, pp. 308-331, 2009.

[5] L. Rong, Z. Feng, and A. S. Perelson, "Mathematical analysis of age-structured HIV-1 dynamics with combination antiretroviral therapy," SIAM Journal on Applied Mathematics, vol. 67, no. 3, pp. 731-756, 2007.

[6] X. Jiang, P. Yu, Z. Yuan, and X Zou, "Dynamics of an HIV-1 therapy model of fighting a virus with another virus," Journal of Biological Dynamics, vol. 3, no. 4, pp. 387-409, 2009.

[7] H. Shu, L. Wang, and J. Watmough, "Sustained and transient oscillations and chaos induced by delayed antiviral immune response in an immunosuppressive infection model," Journal of Mathematical Biology, vol. 68, no. 1-2, 2013.

[8] M. A. Nowak, "Population dynamics of immune responses to persistent viruses," Science, vol. 272, pp. 74-79, 1996.

[9] W. Patrick and A. S Nelson, "A model of HIV-1 pathogenesis that includes an intracellular delay," Mathematical Biosciences, vol. 163, no. 2, pp. 201-215, 2000. 
[10] V. C. Rebecca and S. G. Ruan, "A delay-differential equation model of HIV infection of CD4. T-cells," Mathematical Biosciences, vol. 165, pp. 27-39, 2000.

[11] L. Duan, X. Fang, and C. Huang, "Global exponential convergence in a delayed almost periodic Nicholson's blowflies model with discontinuous harvesting," Mathematical Methods in the Applied Sciences, vol. 41, no. 5, pp. 1954-1965, 2018.

[12] L. Duan, L. Huang, Z. Guo, and X. Fang, "Periodic attractor for reaction-diffusion high-order Hopfield neural networks with time-varying delays," Computers \& Mathematics with Applications, vol. 73, no. 2, pp. 233-245, 2017.

[13] C. Huang, X. Long, and J. Cao, "Stability of antiperiodic recurrent neural networks with multiproportional delays," Mathematical Methods in the Applied Sciences, vol. 43, no. 9, pp. 6093-6102, 2020.

[14] C. Huang, "Stability of almost periodic nicholson's blowflies model involving patch structure and mortality terms," $\mathrm{Ca}$ nadian Mathematical Bulletin, vol. 63, no. 2, pp. 1-20, 2019.

[15] H. Zhu and X. Zou, "Impact of delays in cell infection and virus production on HIV-1 dynamics," Mathematical Medicine and Biology, vol. 25, no. 2, pp. 99-112, 2008.

[16] L. Rong and A. S. Perelson, "Asymmetric division of activated latently infected cells may explain the decay kinetics of the HIV-1 latent reservoir and intermittent viral blips," Mathematical Biosciences, vol. 217, no. 1, pp. 77-87, 2009.

[17] Z. Wang and R. Xu, "Stability and Hopf bifurcation in a viral infection model with nonlinear incidence rate and delayed immune response," Communications in Nonlinear Science and Numerical Simulation, vol. 17, no. 2, pp. 964-978, 2012.

[18] Z. H. Yuan, Z. J. Ma, and X. H. Tang, "Global stability of a delayed HIV infection model with nonlinear incidence rate," Nonlinear Dynamics, vol. 68, pp. 207-204, 2012.

[19] P. W. Nelson and A. S. Perelson, "Mathematical analysis of delay differential equation models of HIV-1 infection," Mathematical Biosciences, vol. 179, no. 1, pp. 73-94, 2002.

[20] M. Y. Li and H. Shu, "Impact of intracellular delays and targetcell dynamics on in vivo viral infections," SIAM Journal on Applied Mathematics, vol. 70, no. 7, pp. 2434-2448, 2010.

[21] M. A. Nowak and C. R. M. Bangham, "Population dynamics of immune responses to persistent viruses," Science, vol. 272, no. 5258, pp. 74-79, 1996.

[22] A. M. Elaiw, "Global properties of a class of virus infection models with multitarget cells," Nonlinear Dynamics, vol. 69, no. 1-2, pp. 423-435, 2012.

[23] P. D. Leenheer and H. L. Smith, "Virus dynamics: a global analysis," SIAM Journal on Applied Mathematics, vol. 63, no. 4, pp. 1313-1327, 2003.

[24] L. Wang and M. Y. Li, "Mathematical analysis of the global dynamics of a model for HIV infection of CD4+ T cells," Mathematical Biosciences, vol. 200, no. 1, pp. 44-57, 2006.

[25] X. J. Yi, S. H. Gong, and L. J. Wang, "Global exponential convergence of an epidemic model with time-varying delays," Nonlinear Analysis Real World Applications, vol. 12, no. 1, pp. 450-454, 2001.

[26] B. Xiao and B. W. Liu, "Exponential convergence of an epidemic model with continuously distributed delays," Mathematical and Computer Modelling, vol. 48, no. 3-4, pp. 541-547, 2008.

[27] L. Duan, M. Shi, C. Huang, and X Fang, "Synchronization in finite-/fixed-time of delayed diffusive complex-valued neural networks with discontinuous activations," Chaos, Solitons \& Fractals, Article ID 110386, 2020, In press.
[28] B. Leonid and B. Elena, "On exponential stability of a linear delay differential equation with an oscillating coefficient," Applied Mathematics Letters, vol. 22, pp. 1833-1837, 2009.

[29] H. Zhang, X. Liu, and M. Yang, "Global exponential stability of a delay reduced SIR model for migrant workers' home residence," Applied Mathematics Letters, vol. 50, pp. 119-125, 2015.

[30] J. Hale and S. M. Verduyn, "Introduction to functional differential equations," in Applied Mathematical ScienceSpringer, New York, NY, USA, 1993.

[31] H. Zhu, X. F. Zou, and X. Zou, "Dynamics of a HIV-1 Infection model with cell-mediated immune response and intracellular delay," Discrete \& Continuous Dynamical Systems$B$, vol. 12, no. 2, pp. 511-524, 2009.

[32] J. Wang, S. He, and L. Huang, "Limit cycles induced by threshold nonlinearity in planar piecewise linear systems of node-focus or node-center type," International Journal of Bifurcation and Chaos, vol. 30, no. 11, Article ID 2050160, 2020.

[33] J. M. Conway and A. S. Perelson, "Early HIV infection predictions: role of viral replication errors," SIAM Journal on Applied Mathematics, vol. 78, no. 4, pp. 1863-1890, 2018.

[34] N. Bellomo, K. J. Painter, Y. Painter, and M Winkler, "Occurrence vs. Absence of taxis-driven instabilities in a may-nowak model for virus infection," SIAM Journal on Applied Mathematics, vol. 79, no. 5, pp. 1990-2010, 2019.

[35] A. M. Elaiw and A. D. Al Agha, "Analysis of a delayed and diffusive oncolytic M1 virotherapy model with immune response," Nonlinear Analysis: Real World Applications, vol. 55, Article ID 103116, 2020. 